\title{
Design of an Acoustic Communication System Based on FHMA for Multiple Underwater Vehicles
}

\author{
Zixin Zhao ${ }^{1}$, Shuxiang Guo ${ }^{2}$ \\ ${ }^{1}$ Graduate School of Engineering, Kagawa University, Hayashi-cho, Takamatsu, Japan; ${ }^{2}$ Department of Intelligent Mechanical Sys- \\ tems Engineering, Kagawa University, Hayashi-cho, Takamatsu, Japan. \\ Email: guo@eng.kagawa-u.ac.jp
}

Received May $13^{\text {th }} 2010$; revised June $20^{\text {th }} 2010$; accepted July $9^{\text {th }} 2010$.

\begin{abstract}
This paper designs an underwater acoustic communication system based on tamed spread spectrum and Frequency Hopping Multiple Access (FHMA) for multiple underwater vehicles. In this system, multiple underwater vehicles can communicate with the console on the water surface simultaneously successfully. The communication system is composed of tamed spread spectrum modulation and demodulation, frequency hopping modulation and demodulation with synchronization function, 4FSK modulation and demodulation and Rake Receiver. In order to make the system more feasible, underwater channel and the effect of user number on Bit Error Ratio (BER) are also taken into account. The simulation results indicate that it is feasible to use this system to accomplish underwater communication reliably for multiple users due to the low BER.
\end{abstract}

Keywords: Underwater Acoustic Communication, Tamed Spread Spectrum, FHMA, Multiple Underwater Vehicles

\section{Introduction}

As more and more attention is paid to the ocean, there are growing interests in exploring and exploiting the ocean these days. In order to realize some tasks such as collection of the information, monitoring of the environment, exploration of energy sources, forecast of natural disaster, assistant navigation in the ocean, underwater communication technology is necessary, which has become a very important scientific research field.

Because acoustic wave is adopted to actualize communication in the water instead of electromagnetic wave, it is called underwater acoustic communication. However, the complex environment in the ocean makes it rather difficult to communicate normally, such as the problems of high noise, multipath effect and large delay. So far, many researches have been made to ensure the communication reliability underwater, including the simulation and measure of the channel [1]; research on the use and algorithm of signal processor in the receiver [2]; diversity reception technique [3] and coding technique (compression coding and error correction coding) [4]. As the tasks that we require underwater vehicles to undertake grow more and more complicated, precise and various, only an underwater vehicle can hardly satisfy all the requirements. Multiple underwater vehicles system becomes one of the most important developmental direc- tions. Our goal is to set up a communication network to ensure the vehicles to work under control normally. At present, multiple access based on spread spectrum technique is necessary to realize communication between multiuser. However, the limitation of frequency bandwidth in underwater acoustic channel results in low rate of original data [5-9]. Some researches focusing on high rate of underwater acoustic communication have been made $[10,11]$, but how to put them to use in the situation of multiuser has not been reported yet. Reference [12] proposed a novel multichannel detection technique based on adaptive multichannel receiver to realize high dada rate in multiuser underwater acoustic communication. But as a whole system, the communication quality was unknown (such as bit error rate) and the near-far effect wasn't taken into account. In order to solve these problems, this paper proposes an underwater acoustic communication system for multiple underwater vehicles. Tamed spread spectrum and FHMA techniques are adopted to deal with the contradiction between the data rate and the limitation of frequency bandwidth. While the data rate is raised, the communication quality still keeps a high level.

This paper is organized as follows: Section 2 analyzes underwater acoustic channel, which is a very important influencing factor in communication system. Section 3 expounds the communication approaches, including the working principle, the components and the structure of 
the system. The simulation results are shown in Section 4. Finally, the conclusions are made and the future research in this field is presented.

\section{Underwater Acoustic Channel}

\subsection{Characteristics of Underwater Acoustic Channel}

Underwater acoustic channel is the most complex communication channel that we have known about. It brings many difficulties to underwater communication. The characteristics of underwater acoustic channel are limitation of frequency bandwidth, varying multipath, fast fading and high noise.

The absorption and the diffusion of acoustic energy relate to transmission distance and frequency. That is to say transmission loss increases as transmission distance and frequency increase. This characteristic results in great attenuation of high frequency signal in long distance transmission. Reference [13] points out that the bandwidth can be lager than $100 \mathrm{kHz}$ in near distance $(<1 \mathrm{~km})$; in middle distance $(1-20 \mathrm{~km})$, the bandwidth is limited in the magnitude of ten kilohertz; the bandwidth is only about several kilohertz in long distance $(20-2000 \mathrm{~km})$.

There are many noise sources in the ocean. Some typical noise sources are listed as the frequency rises [14]. The effect of hydrostatic pressure caused by tide and wave; disturbance of earthquake; onflow; sailing ship; surface wave; thermal noise. For the frequency in the magnitude of ten kilohertz, a main noise source is surface wave. The high noise will cause the original signal difficult to recover.

Because of the reflection of the surface and the floor of ocean as well as the existent of reflectors and scatterers caused by the organisms, acoustic wave will reach the receiving part along several different paths after it is sent. This phenomenon is called multipath transmission. It's a most important factor that affects the performance of underwater acoustic communication. Multipath transmission results in signal distortion (fast fading) and selective fading. The amplitude and the phase of the signal will change along with time and frequency which brings on errors in the receiving part. In order to solve the problem, equalization technique, diversity technique, spread spectrum technique and array technique can be adopted.

\subsection{The Model of Underwater Acoustic Channel}

Because underwater acoustic channel is very complex, it can't be represented by a precise simulation model. Generally speaking, underwater acoustic channel is a kind of slow time-varying coherent multipath channel. In the length of coherent time, it can be simplified as a coherent multipath channel, which only has multipath effect. In this paper, a typical model of acoustic ray is adopted to implement the simulation. The model is expressed as
Figure 1 [15].

When the input signal is $s(t)$, the output signal is

$$
r(t)=\sum_{k=1}^{L} a_{k}(t) s\left(t-\tau_{k}(t)\right)+n(t)
$$

where $L$ is the number of multipath; $a_{k}(t)$ is the timevarying attenuation factor in the $k_{t h}$ transmission path; $\tau_{k}(t)$ is the delay; $n(t)$ is additive white noise.

\section{Communication Approaches}

Because the underwater environment is rather complex, it is very important to decide the structure and the method of communication to ensure the reliability. In this section, the working principle and the structure of the system will be expounded in detail.

\subsection{Tamed Spread Spectrum Communication}

Developing from Direct Sequence Spread Spectrum (DSSS), tamed spread spectrum technique is widely used in communication field in recent years with the characteristics of resisting interference, great security and large capacity. Different with DSSS, tamed spread spectrum accomplishes spread spectrum by way of encoding $(N, k)$. A binary data of $k$ bits is expressed by a sequence of $N$ bits Pseudo Noise (PN) code. The spreading gain of $G=$ $N / k$ is smaller than that of DSSS, and may not be integer, which is very suitable to the limitation of the frequency bandwidth in underwater acoustic communication. There are $2^{k}$ states in a binary data of $k$ bits; accordingly, $2^{k}$ sequences of PN code are necessary. This means that these sequences need to have good characteristics of autocorrelation and cross correlation. In other words, $2^{k}$ sequences of PN code should be orthogonal.

Figure 2 is the model of tamed spread spectrum communication system. The signal after encoded can be expressed as

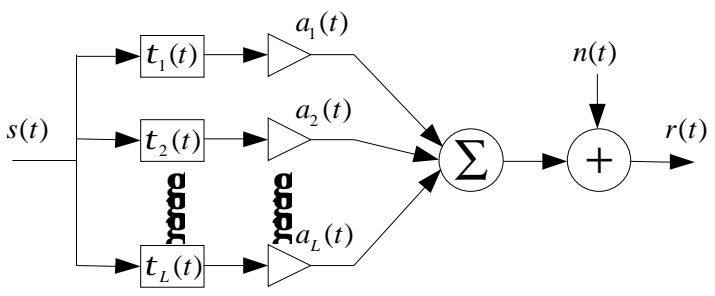

Figure 1. The model of time-varying multipath channel with additive white noise

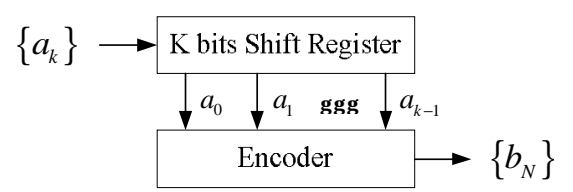

Figure 2. The model of tamed spread spectrum communication system 


$$
b(t)=\sum_{i=0}^{\infty} c_{j}(t-i T)
$$

where $c(t)$ is the PN code, and $j$ is decided by the weight of $a_{k}(t): \quad j=\sum_{n=0}^{k-1} a_{n} 2^{n}, a_{k}(t)=\sum_{m=0}^{k-1} a_{m} g_{a}\left(t-m T_{a}\right), \quad g_{a}(t)$ is gate function, $T=k T_{a}$ is the period of the PN code.

Figure 3 is the framework of the receiver. There are $2^{k}$ paths to execute correlation processing to the $2^{k}$ sequences. Because there is only one correlator that is correlated with the received signal in a period of the PN code, the $k$ bits data in this path can be recovered if the output of the correlator is higher than the threshold, and the demodulation is accomplished.

After correlation processing in the $l_{t h}$ path, the output of the correlator is

$$
y_{l}=\int_{0}^{T} r(t) c_{l}(t) \cos \omega t d t
$$

where $r(t)$ is the input of the correlator, $c_{l}(t)$ is the reference code of the $l_{t h}$ path. $y_{l}$ is composed of signal component and noise component, and the signal component is

$$
y_{l}^{\prime}=\frac{1}{2} \int_{0}^{T} c_{j}(t) c_{l}(t) d t
$$

It is the correlation function of the PN code. If the PN code is orthogonal, the maximum of the autocorrelation value is $T(m=j)$, and the cross correlation value is $0(\mathrm{~m}$ $\neq j)$.

\subsection{FHMA System}

Controlled by the PN code, the carrier frequency of Frequency Hopping (FH) system hops continually and randomly. Compared with DSSS, it has the characteristics of high utilization of the frequency band and solves the problem of near-far effect. They are very important in underwater acoustic communication system, especially in the condition of multiple users.

Figure 4 is the framework of frequency hopping communication system. In the transmitting part, the original signal modulates the carrier produced by the frequency synthesizer, which is controlled by the PN code. After one frequency hopping to anther, it is very difficult to keep phase coherence. Therefore, FSK and ASK that can realize noncoherent detection are adopted as modulation method.

In the receiving part, the signal after processed by the

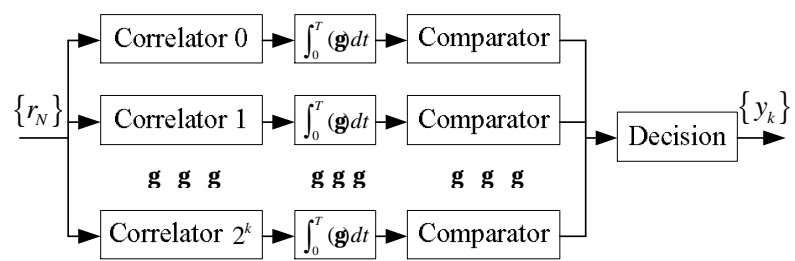

Figure 3. The framework of the receiver of tamed spread spectrum

amplifier will be sent into the mixer. In order to achieve de-spread spectrum of $\mathrm{FH}$, the outputs of the frequency synthesizer should be identical with that in the transmitting part. That is to say, the synchronization of the PN codes in the two parts is necessary. Through mixing, the frequency of the signal will be fixed, which can be demodulated, and the signal is recovered finally. For the undesired signal, it doesn't know the hopping regularity, so the frequency is not correlated with the outputs of the frequency synthesizer in the receiving part. Thus, it can hardly bring on interference to the FH system.

Based on FH, we adopt FHMA to realize underwater acoustic communication simultaneously between multiple users. In the FHMA system, the bandwidth is divided into several channels. The carrier frequency will continually hop along with the time instead of fixed to one channel. The hopping regularity is decided by the PN code of each user. The random hopping of the carrier frequency of each user results in the possibility of multiple accesses in a large frequency extent. In the receiving part, the same as FH, the PN code of each user should keep synchronous with that in the transmitting part. The key point in FHMA system is that the PN code of each user should be mutually orthogonal so as not to affect each other.

\subsection{Receiving Technique of Multipath Signals}

Diversity is a technique which is to reduce the influence of signal fading caused by multipath effect. The basic idea is to divide the received multipath signals into uncorrelated signals, and then to combine the signal energy according to some rule to obtain the energy of the useful signals as much as possible. In this way, the harmful multipath signals are turned into useful signals. The method can maximize the energy of the received useful signals in order to improve Signal to Noise Ratio (SNR).
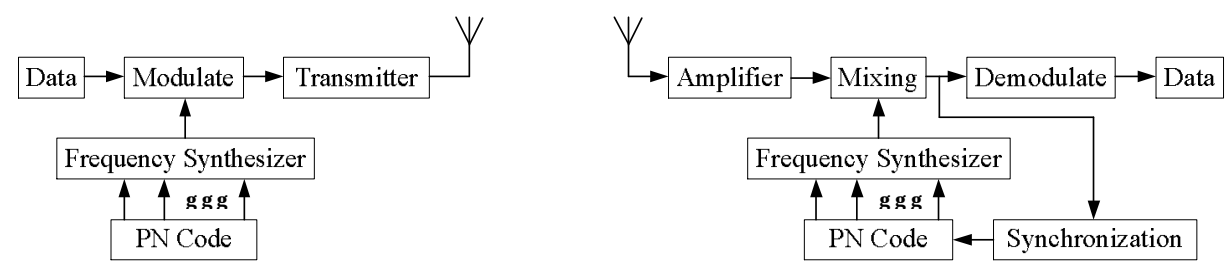

Figure 4. The framework of frequency hopping communication system 
Because of the large multipath delay and the dispersedness of energy in underwater acoustic channel, tap automatic adjusted Rake Receiver is a better choice. Different from tap fixed Rake Receiver, the delay of each correlator in tap automatic adjusted Rake Receiver is adjustable and it is dependent on the estimation made by the multipath searching module. The working principle of Tap automatic adjusted Rake Receiver is shown in Figure 5.

In the figure, the output $z^{\prime}(t)$ is

$$
z^{\prime}(t)=\sum_{i=1}^{L} c_{i}(t) z_{i}(t)
$$

where the weighted coefficient $c_{i}(t)$ is expressed as

$$
c_{i}(t)=z_{i}^{2}(t) / \sum_{n=1}^{L} z_{n}{ }^{2}(t) \quad i=1,2, \mathrm{~L}, L
$$

Maximum ratio combination is selected as combination rule. This method combines the signals based on the SNR of each path. Larger weight is assigned to the path with larger SNR. The SNR and the processing gain $G_{m}$ of the combined signals are as follows:

$$
\begin{aligned}
S N R & =\sum_{i=1}^{L} c_{i} S N R_{i} \\
G_{m} & =L
\end{aligned}
$$

\subsection{Synchronization}

Synchronization is a very important part in a communication system. Only when the receiving part keeps synchronous with the transmitting part, the system can work normally. Many kinds of synchronization need to be considered in a spread spectrum communication system, such as carrier synchronization, bit synchronization and frame synchronization. In this paper, hopping frequency synchronization is mainly discussed.

The requirements of $\mathrm{FH}$ synchronization in underwater acoustic communication include quick establishment, high precision, high reliability and easy realization. According to the requirements and taking the limitation of frequentcy bandwidth into account, we adopted an improved waiting-type self-synchronization method based on matched

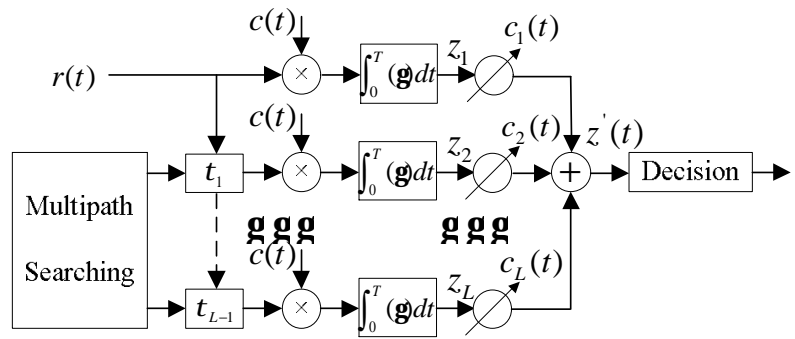

Figure 5. Tap automatic adjusted Rake Receiver

filter. The working principle is shown in Figure 6.

In this method, one matched filter is set to work at the frequency $f$ firstly, waiting for the corresponding frequency $f^{\prime}$ of the FH signal form the transmitting part. When $f^{\prime}$ is detected, the frequency controller will begin to control the matched filter based on the FH pattern. At the same time, synchronization decision is executed. If the received FH sequence is identical with that in the receiving part (decision value is larger than the threshold), that means the synchronization acquisition is successful. The group of matched filters will continue working and the synchronization tracking begins. Otherwise (decision value is smaller than the threshold), the synchronization acquisition is finished by mistake. In this situation, the frequency controller will stop controlling the matched filters and shift $f^{\prime}$ by a chip to the previous frequency point.

The probability density of the synchronization for the first time is

$$
\rho_{1}(t)=\frac{1}{N T_{c}-T_{1}}
$$

where $T_{1}$ is the waiting time for the first time, $N$ is the length of the $\mathrm{FH}$ sequence, $T_{c}$ is the detention time at each $\mathrm{FH}$ point.

If the synchronization fails for the $(n-1) t h$ time, the probability density of the synchronization becomes

$$
\rho_{n}(t)=\frac{1}{N T_{c}-T_{n}}, T_{n}=(n-1) T_{c}+\sum_{m=1}^{n-1} T_{m}
$$

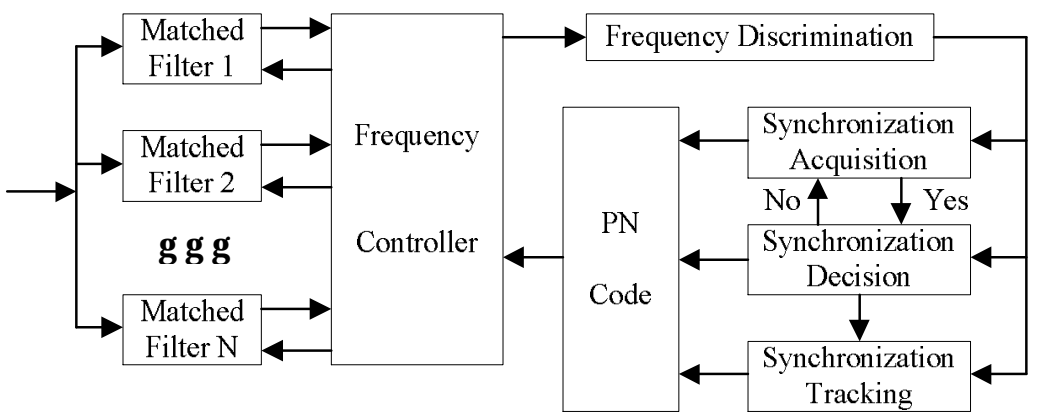

Figure 6. Working principle of the improved waiting-type self-synchronization method 
This equation indicates that the more the times of failure are, the more the probability density of the synchronization is.

For synchronization tracking, a representative tracking technique of FHSS signal is feasible, which is shown in Figure 7 [15].

\subsection{Structure and Model of the Communication System}

The framework of the communication system is shown in Figure 8. It is composed of several underwater vehicles and a console on the surface of water. All the underwater vehicles can communicate with the console simultaneously.

Figure 9 is the principle model of the communication system. In the transmitting part, the original data (digital binary sequence) is tamed spread firstly and then it is 4FSK modulated. Next, the signal is mixed by a series of other frequencies produced by a frequency synthesizer which is controlled by the PN code. Finally, the digital signal is turned into acoustic signal and emitted to the channel through the underwater acoustic transducer. Accordingly, the receiving part is composed of four parts: 1) Rake Receiver; 2) FH de-spread (with synchronization function); 3) 4FSK demodulate; 4) tamed de-spread. Based on the characteristics of underwater acoustic channel combined with FH system, 4FSK noncoherent detection is adopted as demodulation method in this paper.

This section will give the simulation results. The original data and the received data, the frequency spectrogram and the BER curve are included.

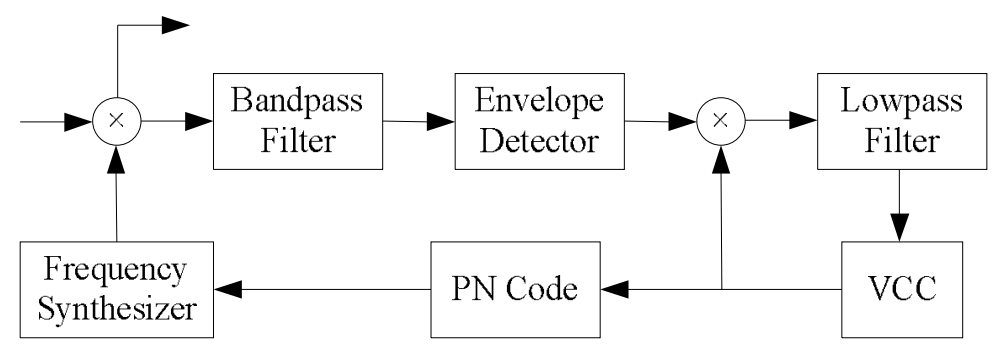

Figure 7. Tracking loop of FHSS signal

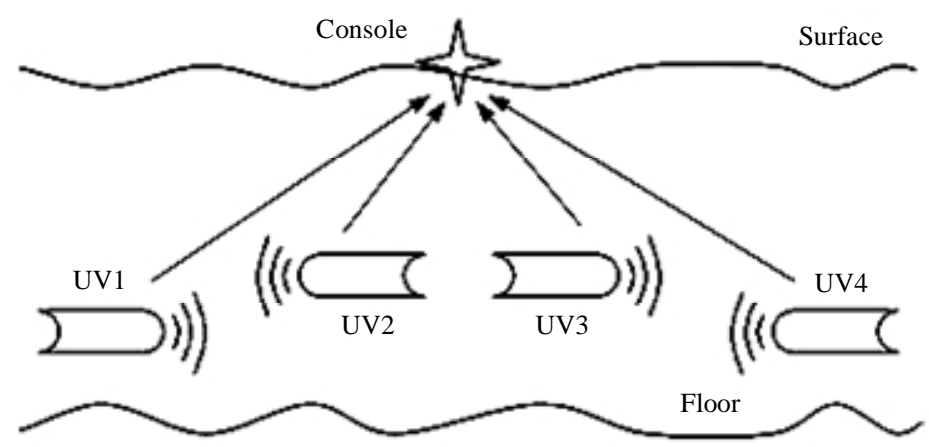

Figure 8. The framework of the communication system

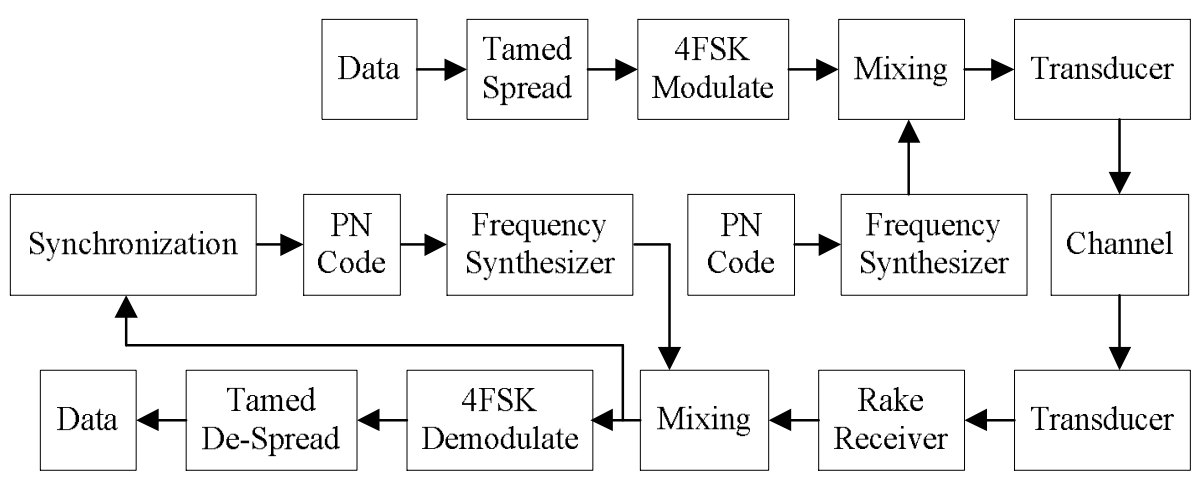

Figure 9. The principle model of the communication system 


\section{Simulation Results}

This section will give the simulation results. The original data and the received data, the frequency spectrogram and the BER curve are included.

\subsection{Design of the Parameters}

In the simulation, the bit rate of original digital sequence is $f_{o}=650 \mathrm{bit} / \mathrm{s}$. Taking the capability and the complexity of the system into account, we use Reed-Solomon (RS) [16] code to realize tame spread spectrum and the parameter of RS code is $(15,5)$. In this situation, the spread factor is 3 and the bit rate after tame spread spectrum is $f_{s}$ $=1.95 \mathrm{kbit} / \mathrm{s}$. In the $4 \mathrm{FSK}$ modulation part, the modulator inputs one of the four frequencies every $T_{f}=1.026 \mathrm{~ms}$, which means the modulator will change the frequency after transmitting every two bits. In order to avoid interference between neighboring channels in FHMA system, the minimum frequency interval should be

$$
\Delta f=n f_{d} \quad(n=1,2,3, \cdots)
$$

where $f_{d}$ is the bandwidth of the signal. In this paper, the interval between the four frequencies of 4FSK is $\Delta f=$ $2 / T_{f}=1.95 \mathrm{kHz}$ and the interval between the frequencyes from the frequency synthesizer in the FH part is $\Delta f^{\prime}=$ $4 \Delta f=7.8 \mathrm{kHz}$. Considering the frequency bandwidth in underwater acoustic communication, we use four frequencies produced by the frequency synthesizer to combine with the four frequencies of 4FSK to form 16 hopping frequencies which is listed in Table 1. The spread factor of FH part is 16, and the total gain of the system is 10

Table1. 4FSK-FH hopping frequencies (Unit: kHz)

\begin{tabular}{cccccc}
\hline & $4 \mathrm{FSK}$ & $f_{1}$ & $f_{2}$ & $f_{3}$ & $f_{4}$ \\
\cline { 2 - 6 } & & 10 & 11.95 & 13.9 & 15.85 \\
\hline$f^{\prime}{ }_{1}$ & 10.4 & 20.4 & 22.35 & 24.3 & 26.25 \\
$f^{\prime}{ }_{2}$ & 18.2 & 28.2 & 30.15 & 32.1 & 34.05 \\
$f^{\prime}{ }_{3}$ & 26 & 36 & 37.95 & 39.9 & 41.85 \\
$f^{\prime}{ }_{4}$ & 33.8 & 43.8 & 45.75 & 47.7 & 49.65 \\
\hline
\end{tabular}

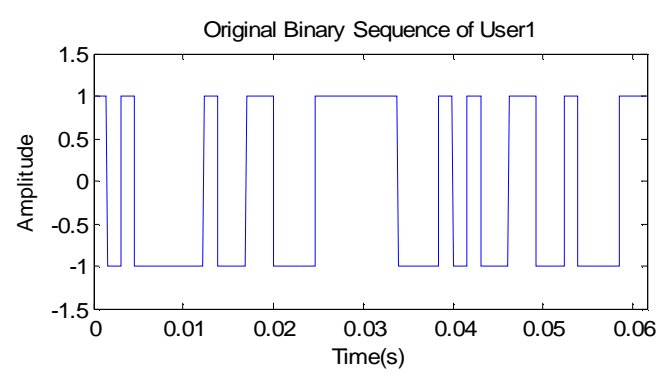

$\log (3 \times 16)=16.8 \mathrm{~dB}$.

In the FH part, Gold code is selected as the PN code. Gold code is a kind of combined codes of $m$ sequence, which is obtained by modular two additions of optimum pairs of two $m$ sequences with the same length, the same rate and the different codes. Its characteristics of autocorrelation and crosscorrelation are very good and the number of available sequences is much more than that of $\mathrm{m}$ sequence. The FH pattern of the system is shown in Figure 10.

The transmission distance is $1 \mathrm{~km}$ from the users to the console on condition that the sound velocity is $1460 \mathrm{~m} / \mathrm{s}$. Also we suppose that there are three transmission paths in the communication channel. One is direct path and the other two are reflection paths. The transmission delay of the two reflection paths are $0.096 \mathrm{~s}$ and $0.168 \mathrm{~s}$ respectively. The transmission losses of the three paths are 9.4 $\mathrm{dB}, 10.7 \mathrm{~dB}$ and $11.7 \mathrm{~dB}$ respectively.

For the convenient of simulation, we suppose there are two users in the system. In fact, because of the good autocorrelation and crosscorrelation of Gold code, the number of users has little influence on the communication effect.

\subsection{Graph of the Signal}

Figure 11 and Figure 12 show the original and the received signal of the communication system. From them we notice that when the SNR is $-10 \mathrm{~dB}$, the original signal can be recovered correctly in the receiving part and the two users can communicate simultaneously and successfully.

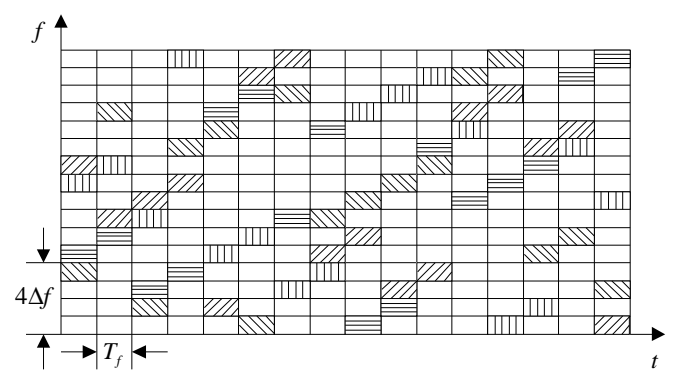

Figure 10. The FH pattern of the communication system

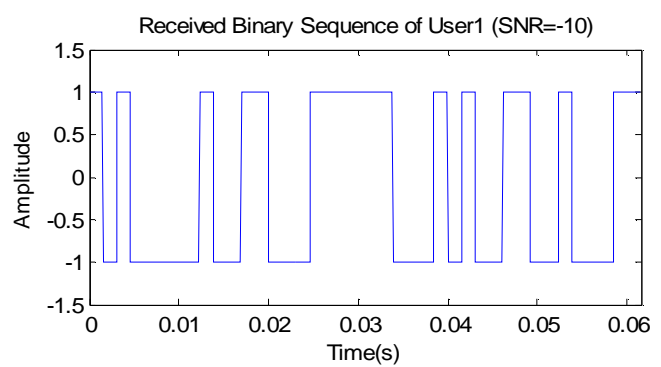

Figure 11. Binary sequence of User 1 

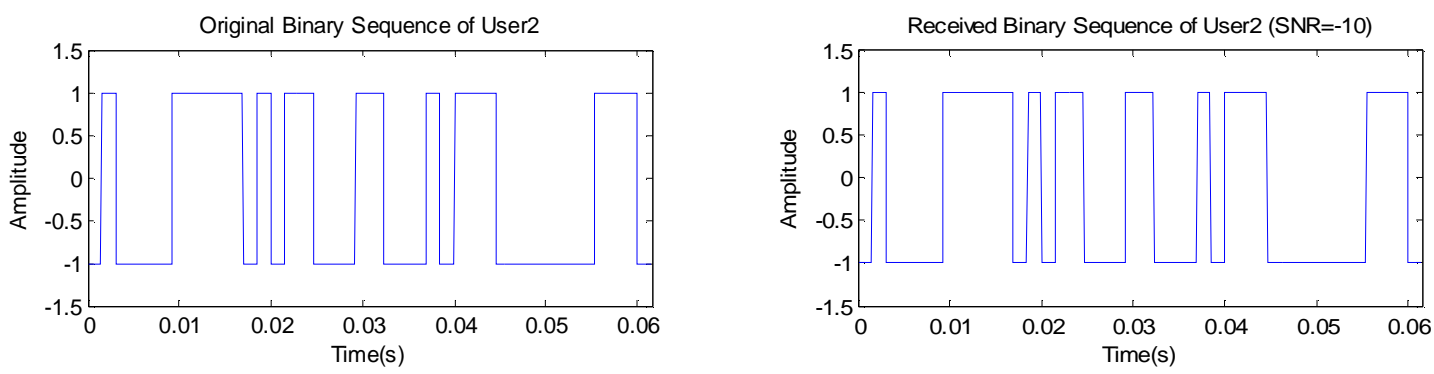

Figure 12. Binary sequence of User 2

Figure 13, Figure 14, Figure 15, Figure 16 show the transforming process of the signal frequency spectrogram (User 1 and User 2) in the transmitting part and the channel. From Figure 13 and Figure 14 we can see that after tamed spread, the frequency spectrogram is spread. Sixteen FH points are shown clearly in Figure 15. Figure 16 indicates that the original signal is submerged in the noise when it is transmitting in the channel. However, due to the adoption of techniques such as spread spectrum, channel coding and Rake receiver, the energy of the noise is reduced in the receiving part and the original signal is recovered.

\subsection{Discussion of BER}

BER is a most important index to estimate the performance of a communication system. In fact, because of the existence of the channel and the noise caused by hardware itself, there are always errors in the communication. What we could do is to reduce the errors to a certain deg-

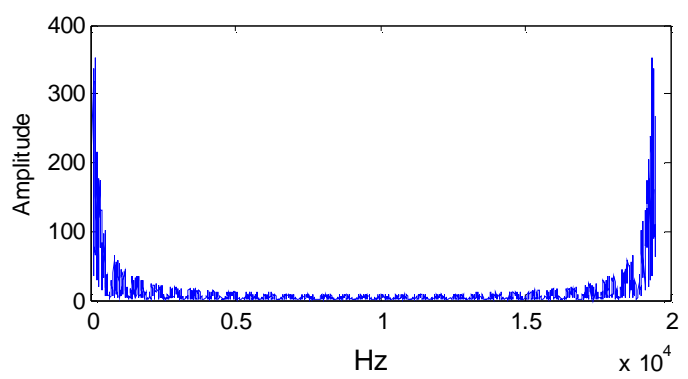

Figure 13. The frequency spectrogram of the original signal

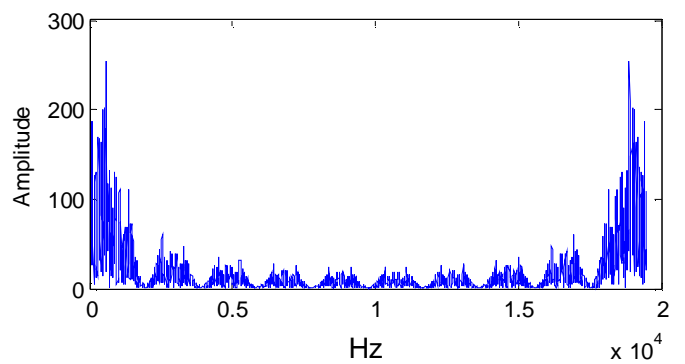

Figure 14. The frequency spectrogram of the signal after tamed spread ree according to the requirement so that they can't affect the basic communication. Generally speaking, in the instruction communication system, the requirement of BER is very strict $\left(<10^{-6}\right)$. Correspondingly, in the voice and image communication system, the requirement of BER is lower $\left(10^{-3}-10^{-5}\right)$. The relationship between BER and SNR of the communication system designed in this paper is shown in Figure 17. From the figure we notice that the BER is less than $10^{-4}$ when the SNR is $-14 \mathrm{~dB}$. The original bit rate is $650 \mathrm{~b} / \mathrm{s}$, it can carry out some data and simple voice communication and the BER level is acceptable in practice.

Figure 18 shows the effect of user number on BER. It indicates that BER grows as user number grows on average because of the interference between each other. But on the whole, the interference is very low due to the good correlation of PN code.

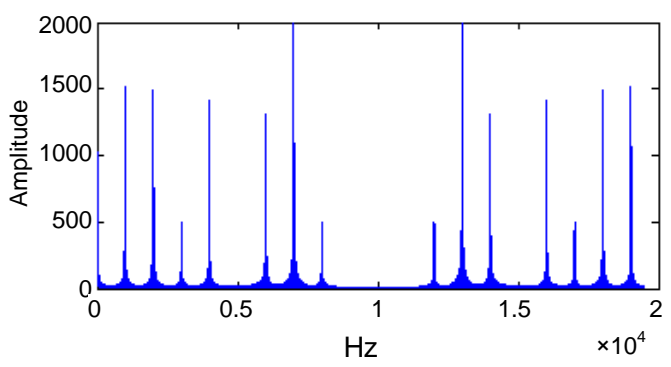

Figure 15. The frequency spectrogram of the signal after FHMA

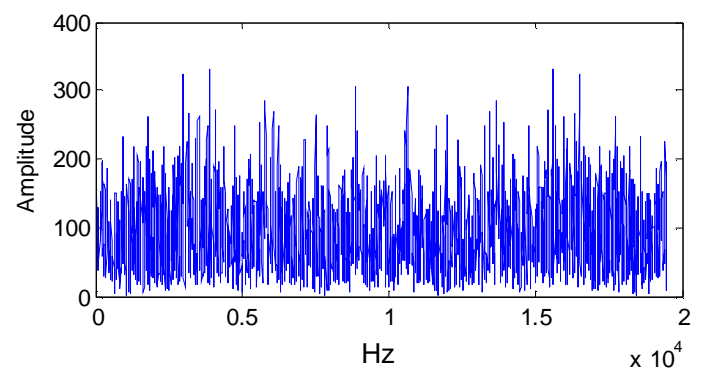

Figure 16. The frequency spectrogram of the signal after adding AWGN 


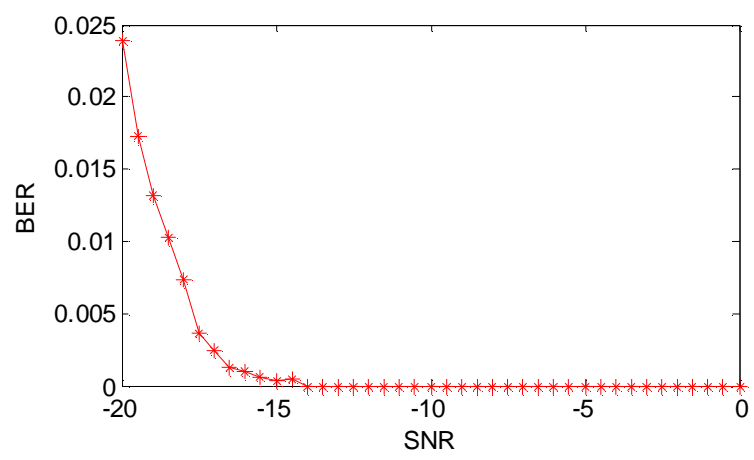

Figure 17. The relationship between BER and SNR

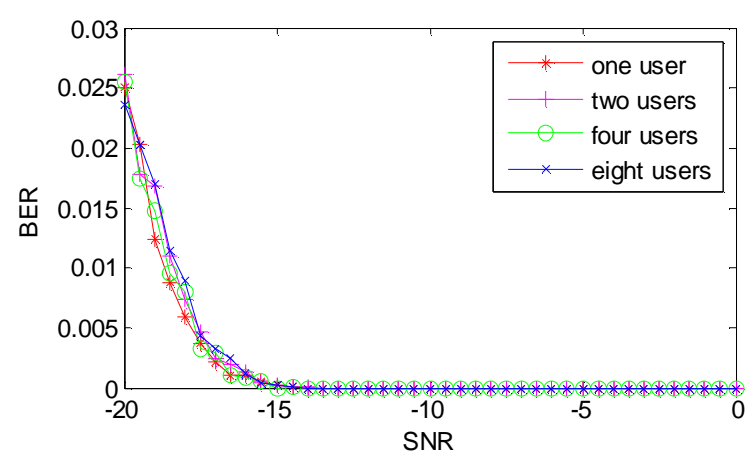

Figure 18. The effect of user number on BER

\section{Conclusions}

This paper designs a kind of underwater acoustic communication system based on tamed spread spectrum and FHMA for multiple underwater vehicles. Aiming at the characteristics of limitation of frequency bandwidth and high noise in underwater acoustic channel, two key techniques of tamed spread spectrum and FHMA are adopted in order to ensure the communication reliability. Besides, Rake Receiver is also an important part in the system to reduce the influence caused by multipath effect. Meanwhile, the synchronization of $\mathrm{FH}$ part and underwater channel are also discussed. The simulation results indicate that this system could enable multiple vehicles to communicate simultaneously successfully. The system could realize some data and simple voice communication, which is acceptable in practice. The theory and the results in this paper have referenced value in the research of underwater acoustic communication.

Next work is to set up duplex communication links between multiple users and the console in order to ensure the equipments can transmit and receive simultaneously at any time, which is a very important problem in the underwater acoustic networks.

\section{Acknowledgements}

This research is supported by Kagawa University Cha- racteristic Prior Research Fund 2010.

\section{REFERENCES}

[1] N. Richard and U. Mitra, "Sparse Channel Estimation for Cooperative Underwater Communications: A Structured Multichannel Approach," IEEE International Conference on Acoustics, Speech, and Signal Processing, Las Vegas, 2008, pp. 5300-5303.

[2] A. Song and M. Badiey, "Generalized Equalization for Underwater Acoustic Communications," Proceedings of MTS/IEEE OCEANS 2005, San Diego, Vol. 2, 2005, pp. 1522-1527.

[3] J.-W. Han, H.-J. Ju, K.-M. Kim, et al., "A Study on the Cooperative Diversity Technique with Amplify and Forward for Underwater Wireless Communication," $O C$ EANS 2008, MTS/IEEE Kobe Techno-Ocean, Kobe, 2008, pp. 1-3.

[4] J. Trubuil, A. Goalic and N. Beuzelin, "A Low Bit-Rate Speech Underwater Acoustic Phone Using Channel Coding for Quality Improvement," Proceedings of IEEE Military Communications Conference MILCOM, Orlando, 2007, pp. 1-7.

[5] T. C. Yang and W.-B. Yang, "Low Signal-to-Noise-Ratio Underwater Acoustic Communications Using DirectSequence Spread-Spectrum Signals," OCEANS 2007Europe, Aberdeen, 2007, pp. 1-6.

[6] C. B. He and J. G. Huang, "Underwater Acoustic Spread Spectrum Communication Based on M Family N group Parallel Transmission," OCEANS 2006-Asia Pacific, Singapore, 2007, pp. 1-4.

[7] X.-Y. Wang, Z.-F. Zhu and S.-L. Fang, "Noncooperative Detection and Parameter Estimation of Underwater Acoustic DSSS-BPSK Signal," 14th International Conference on Mechatronics and Machine Vision in Practice, Xiamen, 2007, pp. 52-56.

[8] S. X. Guo and Z. X. Zhao, "A CDMA Acoustic Communication System for Multiple Underwater Robots," Proceedings of the 2008 IEEE, International Conference on Robotics and Biomimetics, Bangkok, 2008, pp. 15221526.

[9] Z. X. Zhao and S. X. Guo, "A QPSK-CDMA Based Acoustic Communication System for Multiple Underwater Vehicles," Proceedings of the 5th International Conference on Information, Kitakyushu, 2009, pp.124-127.

[10] S.-J. Hwang and P. Schniter, "Efficient Multicarrier Communication for Highly Spread Underwater Acoustic Channels," IEEE Journal on Selected Areas in Communications, Vol. 26, No. 9, 2008, pp. 1674-1683.

[11] S. Roy, T. M. Duman, V. McDonald and J. G. Proakis, "High-Rate Communication for Underwater Acoustic Channels Using Multiple Transmitters and Space-Time Coding: Receiver Structures and Experimental Results," IEEE Journal of Oceanic Engineering, Vol. 32, No. 3, 2007, pp. 663-688.

[12] M. Stojanovic and L. Freitag, "Multichannel Detection for Wideband Underwater Acoustic CDMA Communica- 
tions," IEEE Journal of Oceanic Engineering, Vol. 31, No. 3, 2006, pp. 685-695.

[13] R. J. Vaccaro, "The Past, Present, and Future of Underwater Acoustic Processing," IEEE Signal Processing Magazine, Vol. 15, No. 4, July 1998, pp. 21-51.

[14] R. J. Urick, "Principles of Underwater Sound," 3rd Edition, New York, McGraw-Hill, 1983.
[15] J. G. Proakis, "Digital Communications," 4th Edition MeGraw-Hill Science, New York, 2000.

[16] S. X. Guo and Z. X. Zhao, "Design of a QPSK-CDMA Acoustic Communication System for Multiple Underwater Vehicles," IEEE International Conference on Mechatronics and Automation, Changchun, 2009, pp. 35683572. 